\title{
Adenovirus Ad-p53AIP1-mediated gene therapy and its regulation of $\mathrm{p53}-\mathrm{MDM} 2$ interactions
}

\author{
YUNBO JIANG $^{1 *}$, HUIHUA CHEN ${ }^{1 *}$, HAIQUAN JIA ${ }^{1}$, YUANJI XU ${ }^{1}$, \\ GANG LIU $^{1}$, YAN WANG ${ }^{1}$, XIAOHE YANG ${ }^{2}$ and YINGLIN LU ${ }^{1}$ \\ ${ }^{1}$ Department of Pathobiology, Institute of Basic Medical Sciences, Beijing 100850, P.R. China; \\ ${ }^{2}$ Department of Pathology, University of Oklahoma Health Sciences Center, Oklahoma City, OK 73104, USA
}

Received September 8, 2009; Accepted November 10, 2009

DOI: $10.3892 /$ etm_00000057

\begin{abstract}
We generated replication-defective adenovirus Ad-p53AIP1 and studied its anti-tumor efficacy both in vitro and in vivo. We demonstrated that Ad-p53AIP1 infection elicited high levels of p53AIP1 expression in cancer cells. We also found that Ad-p53AIP1 expression induced marked apoptosis and cell cycle arrest in HepG2 cells. Moreover, Ad-p53AIP1 infection significantly inhibited the tumorigenesis of 4T1 mouse mammary cancer cells in vivo. In particular, we discovered that p53AIP1 overexpression up-regulated the protein levels of p53 in HepG2 cells, which was accompanied by down-regulation of MDM2 mRNA and protein, suggesting an interaction between MDM2 and p53 in p53AIP1-induced apoptosis and cell cycle arrest. Our data demonstrated the feasibility of Ad-p53AIP1-mediated cancer gene therapy. p53AIP1-induced up-regulation of $\mathrm{p} 53$ protein through MDM2 suggests that p53AIP1 gene therapy may be more advantageous in tumors expressing high levels of oncoprotein MDM2 or having a mutation in MDM2 inhibitor p16INK4.
\end{abstract}

\section{Introduction}

The functional p53 pathway is critical for homeostasis and tumor inhibition. p53 mutations, which are detected in more than half of human cancers, have been associated with tumor development, poor prognosis and therapeutic resistance. Various therapeutic strategies aiming to rescue or amplify p53 pathways are being developed for cancer treatment (1-3). In

Correspondence to: Dr Yinglin Lu, Department of Pathobiology, Institute of Basic Medical Sciences, 27 Taiping Road, Beijing 100850, P.R. China

E-mail: luyinglin@hotmail.com

Dr Xiaohe Yang, Department of Pathology, University of Oklahoma Health Sciences Center, Oklahoma City, OK 73104, USA

E-mail: xiaohe-yang@ouhsc.edu

*Contributed equally

Key words: p53AIP1, p53, MDM2, cell cycle, apoptosis, gene therapy particular, p53AIP1 is emerging as a therapeutic target with great potential.

The p53AIP1 gene is a p53 target gene located on chromosome 11. Alternative splicing gives rise to three isoforms $(\alpha, \beta$ and $\gamma$ ) of p53AIP1 proteins (4). Functional studies indicate that p53AIP1 is a potent pro-apoptotic molecule. Overexpression of p53AIP1 alone induces apoptosis (5). Blockage of p53AIP1 using specific siRNA significantly attenuates p53-induced apoptosis (4). Available data suggest that p53AIP1 induces apoptosis through the mitochondrial pathway. By interacting with Bcl-2, p53AIP1 decreases mitochondrial membrane potential and induces cytochrome c release, which leads to caspase-9 activation and the downstream activities (6).

Expression of p53AIP1 is primarily regulated by p53. Activation of p53 by a number of factors, such as DNA damage, induces the transcription of p53AIP1 (7-10). In contrast to the p53-mediated regulation of p21 and MDM2, which involves the phosphorylation of Ser-15 of p53 $(11,12)$, induction of p53AIP1 mRNA and its apoptotic function was uniquely associated with the phosphorylation of Ser-46 of p53 (4). Substitution of Ser-46 impairs p53-mediated apoptosis and its induction of p53AIP1 transcription.

The clinical relevance of p53AIP1 is supported by reports on its association with prognosis and therapeutic potential. Sawaya et al reported that p53AIP1 mRNA levels were significantly lower in gastric carcinoma cells, as compared to chronic gastritis samples (13). Data by Yamashita et al revealed that p53AIP1 gene expression in a lymph node metastasis-positive group was significantly lower than in a negative group, and the overall survival of a p53AIP1 low expression group was significantly worse than that of a p53AIP1 high expression group (14). A study by Yoshida et al indicated that Ad-p53AIP1 induced apoptosis in a number of cancer cell lines. It appeared that cells with wild-type p53 were more sensitive to Ad-p53AIP1 than cells with mutant p53 (5). However, the mechanism of p53-related sensitivity was not elucidated.

In this study, we generated Ad-p53AIP1 and established in vitro and in vivo models to evaluate Ad-p53AIP1-induced tumor inhibition. We characterized Ad-p53AIP1-induced apoptosis and cell cycle arrest in HepG2, HeLa and 4T1 cells. Our data demonstrated that p53AIP1-induced up-regulation of p53 through MDM2 down-regulation is an important 
mechanism underlying Ad-p53AIP1-induced apoptosis and cell cycle arrest.

\section{Materials and methods}

Reagents. Plasmids pDC316 and pBHGlox $\Delta \mathrm{E} 1,3$ Cre were purchased from Fungenome Technology Co., Ltd. and Vector Gene Technology Co., Ltd., respectively. Ad-null viruses were a gift from Vector Gene Technology Co., Ltd. Antibodies against p53AIP1, $\beta$-actin, p21, p53 and MDM2 were purchased from Santa Cruz Biotechnology, Inc. Anti-cleaved PARP antibody was purchased from Cell Signaling Technology, Inc. Anti-CDK4 antibody was purchased from Lab Vision Inc., Fremont, CA. Rhodamine 123 was from Sigma Co., and the reverse transcription kit was purchased from Jingmei Biotech Co., Ltd.

Construction, characterization and amplification of p53AIPIencoding adenovirus. The p53AIP1 gene was cloned from a cDNA library using the following primers: forward, 5 ATG GGA TCT TCC TCT GAG GCG A 3'; reverse, 5' TCA CTG CAA CCT CAA CGG TGC T 3'. The cloned gene was verified by DNA sequencing. The p53AIP1 gene was then subcloned into the pDC316 shuttle vector. For adenovirus packaging, pDC316-p53AIP1 and pBHGlox $\Delta \mathrm{E} 1,3 \mathrm{Cre}$ (the backbone vector of the AdMax adenoviral vector system) were co-transfected into HEK293 cells using Lipofectamine 2000 (Invitrogen). Twelve days after transfection, individual plaques were chosen for DNA purification. p53AIP1 integration was examined by PCR using the above mentioned primers. Viruses derived from p53AIP1-positive clones were named Ad-p53AIP1. This virus was then amplified and titrated as described previously (15).

Cell culture and viral transduction. Human hepatocellular carcinoma cell line HepG2, human embryonic kidney cell line HEK293 and murine BALB/c derived mammary tumor cell line 4T1 cells were cultured in H-DMEM medium supplemented with $10 \%$ FBS and antibiotics. For viral transduction, unless specified, the cells were infected with Ad-null or Ad-p53AIP1 at a MOI of 100 for $24 \mathrm{~h}$, followed by specific examinations.

RT-PCR. To examine the mRNA levels of p53AIP1, p53 and MDM2 in HepG2 cells, total RNA was extracted from the cells 6,12 and $24 \mathrm{~h}$ after transduction. First-strand cDNA was synthesized using a kit from Jingmei BioTech Co. Ltd., following the manufacturer's protocol. The primer sequences for the PCR reactions were as follows: p53 forward, 5 ' AGC ACT GTC CAA CAA CAC CA 3'; reverse, 5' TCC ATG TCA GTC TGA GTC AG 3'; MDM2 forward, 5' CCC TGG TTA GAC CAA AGC CAT 3'; reverse, 5' GGC ACG CCA AAC AAA TCT CC 3'. The PCR conditions were $94^{\circ} \mathrm{C} / 5 \mathrm{~min}$, $\left(94^{\circ} \mathrm{C} / 30 \mathrm{sec}, 53^{\circ} \mathrm{C} / 30 \mathrm{sec}, 72^{\circ} \mathrm{C} / 30 \mathrm{sec}\right) 32$ cycles, $72^{\circ} \mathrm{C} / 7 \mathrm{~min}$. PCR products $(20 \mu \mathrm{l})$ were electrophoresed on a $2 \%$ agarose gel.

Western blotting. Cell lysates were extracted from the treated cells at indicated time-points after viral transduction, followed by protein concentration determination. The protein lysate $(50 \mu \mathrm{g})$ from each sample was separated using $10 \%$ SDS-PAGE gel and transferred to a nitrocellulose membrane. The membrane was blocked with 5\% milk and probed with the primary antibody against the protein to be detected. The specific bands were visualized with ECL.

Effect of p53AIP1 on cell survivallgrowth by the 3-(4, 5-dimethylthiazol-2-yl)-2, 5-diphenyltetrazolium bromide (MTT) assay. Two thousand cells were plated into each well of 96-well plates one day prior to viral transduction. The cells were then transduced with Ad-null or Ad-p53AIP1 virus at a MOI of 100 for $4 \mathrm{~h}$, followed by medium replacement. Using the MTT assay, cell survival/proliferation was determined daily after treatment for 5 days. For MTT assay, the cells were incubated with MTT $(500 \mu \mathrm{g} / \mathrm{ml})$ for $3 \mathrm{~h}$. Incorporated MTT was dissolved and measured at $D_{n m} 570$. Each data set was based on six parallel samples.

Flow cytometric detection of cell cycle distribution and mitochondrial membrane potential. For cell cycle analysis, the treated cells were trypsinized and collected at indicated times, followed by fixation with paraformaldehyde. Fixed cells were washed with PBS containing $0.1 \%$ Triton X-100 and treated with RNase A for 30 min and stained with $50 \mu \mathrm{g} / \mathrm{ml}$ propidium iodide. Cells were analyzed using a FACS Calibur flow cytometer. Cell cycle distribution was analyzed using MultiFit software. To measure the mitochondrial membrane potential, treated cells at indicated time points were typsinized, pelleted and washed with PBS. Cells in suspension were then stained with Rhodamine $123(5 \mu \mathrm{g} / \mathrm{ml})$ in the dark for $30 \mathrm{~min}$, followed by flow cytometric analysis.

Transmission electron microscopy. Non-treated control and Ad-null or Ad-p53AIP1 transduced (100 MOI for $96 \mathrm{~h}$ ) HepG2 cells were fixed in $2.5 \%$ glutaraldehyde for $4 \mathrm{~h}$ then treated with $1 \% \mathrm{OsO}_{4}$. The samples were then dehydrated through a graded ethanol series and embedded, followed by ultra-thin sectioning, staining and viewing under an electron microscope.

Hoechst staining. The cells treated with either Ad-null or Ad-p53AIP1 at 100 MOI for $48 \mathrm{~h}$ were collected (including floating and trypsinized cells) and washed with PBS followed by fixation with $2 \%$ paraformaldehyde at $4^{\circ} \mathrm{C}$ for $30 \mathrm{~min}$. The cells were stained with $0.5 \mu \mathrm{g} / \mathrm{ml}$ Hoechst 33258 for $30 \mathrm{~min}$. Stained cells were then washed and mounted on slides using a cytospinner. Apoptotic cells with nuclear condensation or fragmentation were counted under an Olympus fluorescence microscope.

In vivo tumor xenograft study. Female BALB/c mice (6 weeks old, 18-22 g) and murine mammary tumor cell line 4T1 were used for this study. The first set of experiments was designed to examine in vivo tumor development of $4 \mathrm{~T} 1$ cells transduced with Ad-p53AIP1 prior to implantation. With 7 mice in each group, the groups included PBS treatment, Ad-null transduction and Ad-p53AIP1 transduction. After exposure to PBS or Ad-null or Ad-p53AIP1 virus in vitro for $24 \mathrm{~h}$, 4T1 cells from each group were injected s.c. into the flanks of the mice $\left(1 \times 10^{6}\right.$ cells $/ 100 \mu \mathrm{l} /$ mouse). After transplantation, 
A

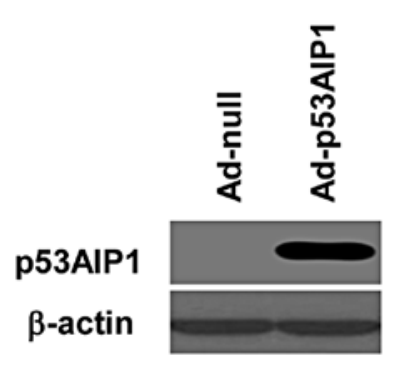

B
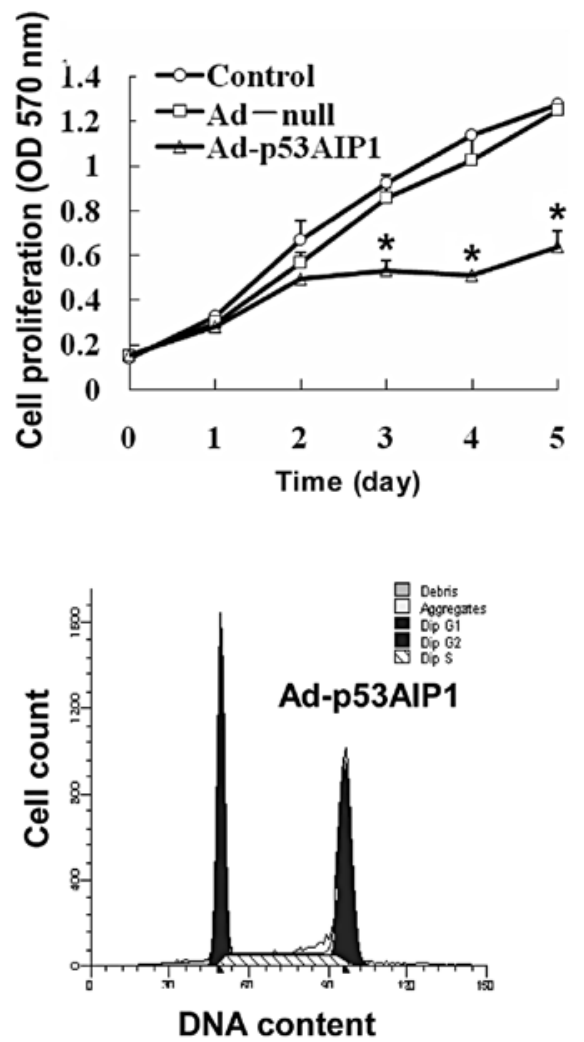

Figure 1. Adenovirus-mediated overexpression of p53AIP1 induces growth inhibition and cell cycle arrest. (A) Western blot detection of p53AIP1 expression in HepG2 cells transduced with Ad-null and Ad-p53AIP1. (B) MTT assay determination of Ad-p53AIP1-mediated growth inhibition in HepG2 cells; *p<0.01. (C) Flow cytometric analysis of cell cycle distribution of HepG2 cells 24 h after Ad-null or Ad-p53AIP1 treatment.

tumor size was measured using calipers, and tumor volume was estimated according to the following formula: Tumor

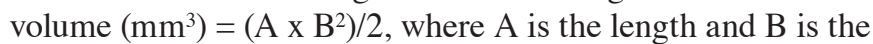
tumor width. Tumor inhibition rate $(\%)=[$ (average of control tumor volume - average of Ad-p53AIP1 tumor volume)/ average of control tumor volume] x $100 \%$. The second set of experiments involved examining the effect of in vivo delivery of Ad-p53AIP1 on 4T1 tumor development. To this end, untreated 4T1 cells were implanted into 21 mice at a dose of $1 \times 10^{6}$ cells $/ 100 \mu \mathrm{l} /$ mouse. The mice were randomly divided into three groups when tumor volumes reached $4-5 \mathrm{~mm}$ in diameter. With 7 mice in each group, the animals were treated with a multi-spot intra tumor injection of PBS, Ad-null or Ad-p53AIP1 at $2 \times 10^{8} \mathrm{PFU} / 100 \mu \mathrm{l} /$ mouse. The injections were repeated two more times with an interval of 7 days. Tumor development and documentation were performed as above.

Statistical analysis. Data were presented as the mean \pm SD. Statistical analysis was performed using SAS 8.0 software package.

\section{Results}

Expression of p53AIP1 in Ad-p53AIP1-transduced HepG2 cells. We first constructed the Ad-p53AIP1 virus to deliver p53AIP1 expression in cancer cells. We then examined the protein levels of p53AIP1 in HepG2 cells transduced with Ad-null or Ad-p53AIP1. Compared to non-detectable p53AIP1 in mock-transduced cells, the cells transduced with
Ad-p53AIP1 expressed high levels of p53AIP1 (Fig. 1A), which provided a solid foundation for further functional studies.

To test the effect of Ad-p53AIP1 transduction on cell proliferation, we treated HepG2 cells with 100 MOI of Ad-null or Ad-p53AIP1 virus and compared their proliferation rates with the non-treatment control. As shown in Fig. $1 \mathrm{~B}$, the proliferation of Ad-p53AIP1-transduced cells was significantly inhibited, which was approximately one half of the control cells on day 5. To test whether Ad-p53AIP1 has any effect on cell cycle progression, we analyzed the cell cycle distribution of HepG2 cells infected with Ad-p53AIP1 for 24 h. We found that p53AIP1 expression increased the number of HepG2 cells in G2/M phases. Since this was accompanied by decreased proliferation, the data suggest that Ad-p53AIP1 induced G2/M arrest in HepG2 cells, which contributed to its growth inhibition effect (Fig. 1C).

p53AIP1 expression induces apoptosis in HepG2 cells. As p53AIP1 is a pro-apoptotic molecule, we characterized Ad-p53AIP1-induced apoptosis in HepG2 cells by poly(ADPribose) polymerase (PARP) cleavage, electron microscopy, mitochondrial membrane potential and Hoechst staining. As shown in Fig. 2A, in contrast to non-detectable cleavage on Ad-null infected cells, PARP cleavage was readily detected in HepG2 cells infected with Ad-p53AIP1 for $48 \mathrm{~h}$. Under a transmission electron microscope (Fig. 2B), apoptotic HepG2 cells induced by Ad-p53AIP1 infection for $96 \mathrm{~h}$ displayed chromatin condensation with clumped perinuclear distribution 
A

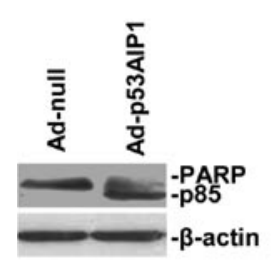

B

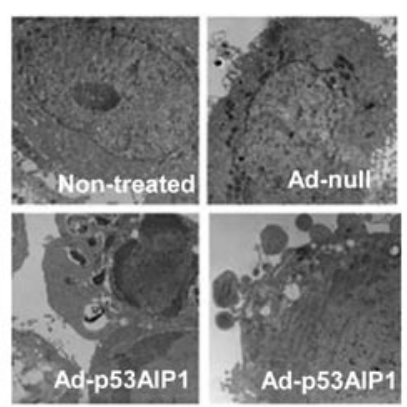

C
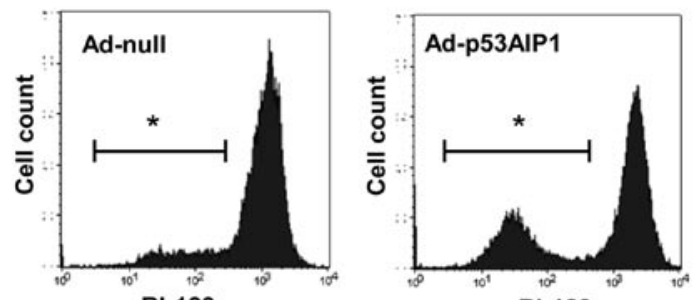

Rh123

D

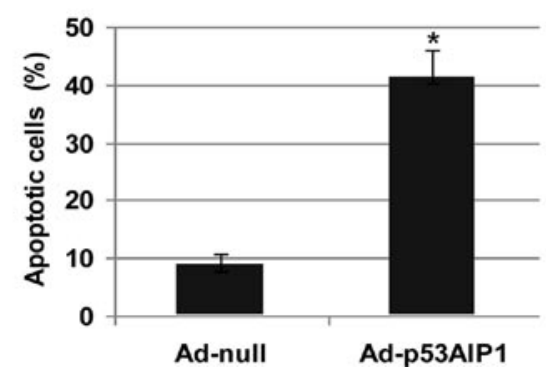

Figure 2. Ad-p53AIP1-mediated apoptosis in HepG2 cells. (A) Western blot detection of PARP cleavage, as indicated by the decrease in the intact protein and the appearance of the p85 subunit. (B) Ultrastructural change in HepG2 cells infected with Ad-p53AIP1 by transmission electron microscope observation $(x 6,000)$. (C) Flow cytometric analysis of mitochondrial membrane potential $(\Delta \psi \mathrm{m})$ of Ad-null- or Ad-p53AIP1-infected HepG2 cells stained with Rhodamine 123. "Cells with low mitochondrial membrane potential were considered to undergo apoptosis. (D) Apoptosis detected by Hoechst staining. HepG2 cells treated with the indicated virus for $48 \mathrm{~h}$ in triplicate were stained with Hoechst 33258. Cells with nuclear condensation or fragmentation were counted as apoptotic cells; ${ }^{*} \mathrm{p}<0.01$.

and nuclear fragmentation. Other features included membrane blebbing, loss of microvilli and appearance of apoptotic bodies.

Flow cytometric analysis of mitochondrial membrane potential with Rhodamine 123 revealed that the fluorescent intensity in Ad-p53AIP1-infected HepG2 cells decreased as infection time increased. In contrast to a geometric mean value of 889.40 in Ad-null-infected cells, the fluorescent intensity decreased to 502.64 in the Ad-p53AIP1-infected cells $72 \mathrm{~h}$ after infection, suggesting that p53AIP1-induced apoptosis in HepG2 cells involves mitochondrial membrane potential collapse (Fig. 2C). Moreover, nuclear staining with Hoechst 33258 showed that nuclear condensation and/or fragmentation were detected in $41 \%$ of Ad-p53AIP1-induced cells, as compared to a 9\% change in Ad-null-infected cells (Fig. 2D). In addition, we also observed similar apoptotic patterns in HeLa cells infected with Ad-p53AIP1 (data not shown). Taken together, these data demonstrated that Ad-p53AIP1 induced potent apoptotic activity in the infected cells.

A

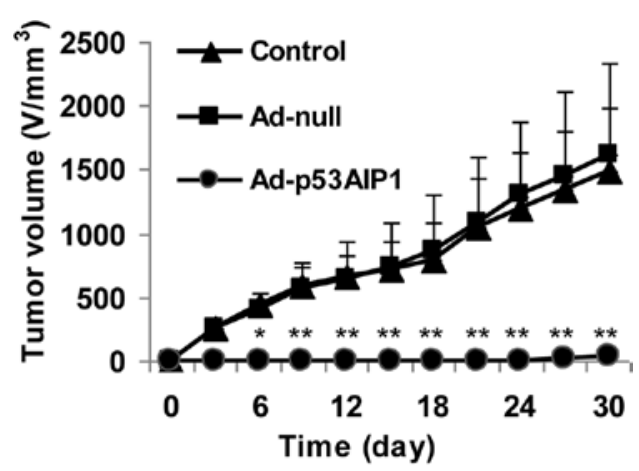

B

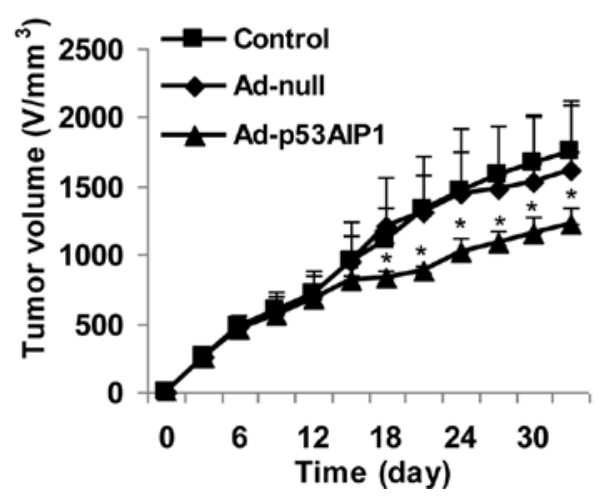

Figure 3. Ad-p53AIP1-mediated inhibition of the 4T1 mouse tumor cell line in vivo. (A) Effect of Ad-p53AIP1 on tumor development of 4T1 cells treated with Ad-p53AIP1 before implantation. (B) Effect of in vivo delivery of Ad-p53AIP1 on 4T1 tumor development. ${ }^{* *} \mathrm{p}<0.01$; ${ }^{*} \mathrm{p}<0.05$, as compared to the Ad-null group at the same time-point.

Ad-p53AIP1 inhibits the development and growth of $4 T 1$ breast tumors in vivo. We next examined the efficacy of Ad-p53AIP1 on tumor growth in vivo using a 4T1 breast tumor model. In the first set of experiments, 4T1 tumor cells were treated with control (PBS), Ad-null or Ad-p53AIP1 virus for $24 \mathrm{~h}$ before implantation. The results showed that the tumors in the control and Ad-null groups grew progressively, reaching $\sim 1,700 \mathrm{~mm}^{3}$ by day 30 . In contrast, tumor growth was minimal in the group treated with Ad-p53AIP1. The tumors were not palpable until day 21 (Fig. 3A). The striking difference between the control and Ad-p53AIP1-treated tumors indicates that Ad-p53AIP1 has a potent tumor inhibition effect. In the second set of experiments, we tested the effect of Ad-p53AIP1 on the progression of 4T1 tumors. After the tumors were palpable, $2 \times 10^{8}$ PFU Ad-null or Ad-p53AIP1 viruses were administered to the tumors by multi-spot intratumor injection. As shown in Fig. 3B, tumor growth in the Ad-p53AIP1-treated group was significantly slower $(\mathrm{p}<0.05)$ than in the control and the Ad-null groups.

Up-regulation of p53 in Ad-p53AIPI-transduced cells. To study the underlying mechanisms of p53AIP1 overexpressioninduced growth inhibition and apoptosis, we examined the expression of a number of key factors involved in apoptosis and cell cycle regulation, including p53, p21, Bax, Bcl-2 and CDK4 in HepG2 cells. In contrast to minimal changes in Ad-null-infected cells (data not shown), we found that p53 protein levels were significantly up-regulated after 
A

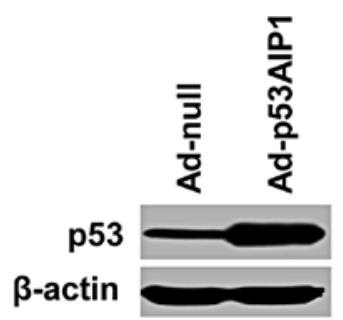

B

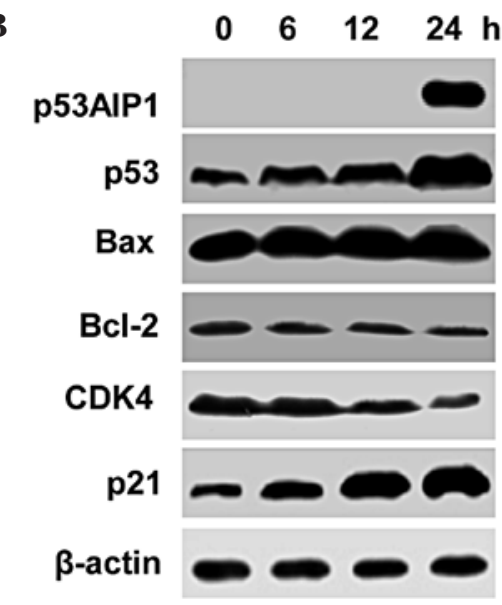

C

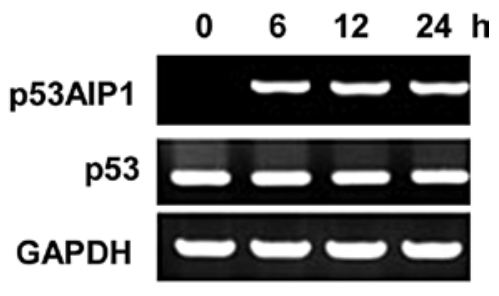

D

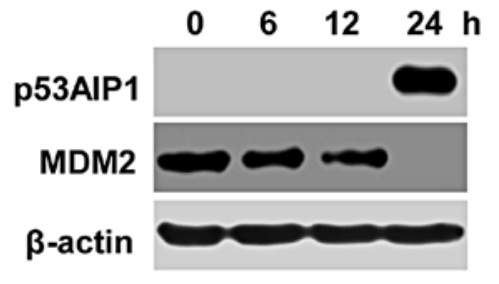

E

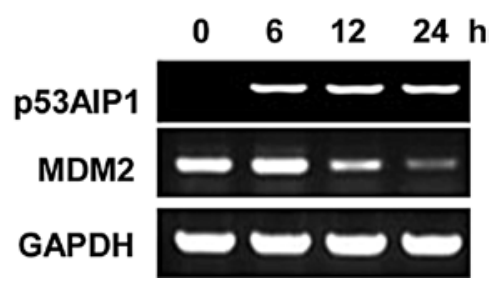

Figure 4. p53AIP1 expression induces p53 up-regulation through down-regulation of MDM2. (A) p53 protein levels in HepG2 cells infected with Ad-p53AIP1. (B) Western blot detection of p53AIP1, p53, Bax, Bcl-2, CDK4 and p21 in Ad-p53AIP1-infected HepG2 cells at different time points. (C) mRNA levels of p53AIP1 and p53 in Ad-p53AIP1-infected HepG2 cells detected with RT-PCR. (D) Protein levels of p53AIP1 and MDM2 in Ad-p53AIP1-infected HepG2 cells. (E) mRNA levels of p53AIP1 and MDM2 in Ad-p53AIP1-infected HepG2 cells detected with RT-PCR.

Ad-p53AIP1 infection (Fig. 4A), which was accompanied by a significant increase in p21, a moderate increase in Bax and decrease in Bcl-2 and CDK4 (Fig. 4B). Since p53 mRNA levels in Ad-p53AIP1-transduced cells were not increased (Fig. 4C), p53AIP1-induced up-regulation of p53 appears to occur at the protein level rather than the transcriptional level. These results suggest that p53AIP1 not only functions as a target of p53 but also has a feedback action on p53 expression.

Ad-p53AIP1 down-regulates MDM2 transcription. To elucidate the mechanism of p53AIP1-mediated up-regulation of p53 protein levels, we examined whether the expression of MDM2, a p53-specific E3 ubiquitin ligase, was affected by p53AIP1 expression in HepG2 cells. We found that both MDM2 protein and mRNA levels were decreased in Ad-p53AIP1-transduced cells (Fig. 4D and E). A decrease in MDM2 and a corresponding increase in p53 expression in p53AIP1-overexpressing cells suggest that p53AIP1 up-regulates p53 through down-regulation of MDM2, which leads to increased p53 stability.

\section{Discussion}

Gene therapy targeting p53 pathways is a promising new approach in cancer treatment. Adenovirus-mediated p53 gene therapy for human cancers has been approved for clinical application in China (16). However, direct delivery of p53 might be compromised by certain p53 antagonists in cancer cells, such as MDM2 overexpression $(17,18)$. Thus, identifying novel targets to rescue or augment the function of the p53 pathway has been actively pursued. In this study, we tested the effects of Ad-p53AIP1 on cancer cell proliferation and apoptosis in both in vitro and in vivo settings. We also discovered a novel mechanism of p53AIP1-induced tumor regression.

p53AIP1 is a p53 target gene and a potent pro-apoptotic molecule. Since it is downstream of p53 and can circumvent p53 compromising factors, these characteristics make it a good candidate for a p53 alternative in cancer gene therapy. Recent association between low levels of p53AIP1 in human cancer tissues and a poor prognosis further supports its implication in cancer therapy (4). Previously, Yoshida et al examined the Ad-p53AIP1-mediated apoptotic effect on a number of cancer cell lines and found that p53AIP1 alone was able to induce marked apoptosis in many cell lines. Ad-p53AIP1 appeared to be more effective in $\mathrm{p} 53^{+/+}$cells, although the mechanism was not clear (5).

In this study, we constructed Ad-p53AIP1 and established its in vitro and in vivo models for further studies, especially in mechanistic investigation. Using HepG 2 cells, we demonstrated that Ad-p53AIP1, not only stimulates apoptosis, but also induces cell cycle arrest in G2/M phases. p53AIP1-induced cell cycle arrest was accompanied by p21 up-regulation and CDK4 down-regulation. This novel finding suggests that p53AIP1 may function beyond apoptotic induction and that its cell cycle regulation may also contribute to p53AIP1-mediated tumor regression. Moreover, data from in vivo experiments demonstrated that Ad-p53AIP1 effectively inhibited 4T1 tumor development and progression. Ad-p53AIP1-mediated tumor regression was more effective using pre-inoculation treatment than intra-tumor injection. This can partially be explained by the low efficiency associated with in vivo viral delivery. 
One of the major findings of this study is that p53AIP1 may up-regulate p53 protein levels in HepG2 cells. We demonstrated that p53AIP1 expression up-regulated p53 protein levels but not mRNA levels. We also found that p53AIP1 down-regulated MDM2 transcription. Since MDM2 is a ubiquitin ligase that induces p53 degradation (19-21), p53AIP1-mediated inhibition of MDM2 might lead to p53 protein accumulation. This finding may partially explain why p53AIP1 is more effective in cancer cells with wild-type p53 (5) and why cell cycle regulation was modified in p53AIP1overexpressing cells (Fig. 2). However, p53AIP1-induced up-regulation of p53 and down-regulation of MDM2 in HeLa cells were less evident when compared to HepG2 cells (data not shown). Hence, the factors that determine p53AIP1induced MDM2-p53 interaction and cause cell line-dependent discrepancy require further investigation.

In summary, we established in vitro and in vivo models with which to study Ad-p53AIP1-mediated tumor inhibition. These can be used to explore more specific factors that may affect the efficacy of Ad-p53AIP1-induced apoptosis and cell cycle arrest. Our findings related to p53AIP1-induced MDM2-p53 interaction in HepG2 cells are of great significance. They suggest that gene therapy targeting Ad-p53AIP1 may be more advantageous for tumor cells overexpressing oncoprotein MDM2 or having a mutation in the MDM2 inhibitor p14ARF and p16INK4 $(22,23)$. Further studies along this line may open a new avenue for fighting human cancers.

\section{Acknowledgements}

This study was supported by grants from the Major State Basic Research Development Program of China (to Dr Yinglin Lu, no. 2002CB513105).

\section{References}

1. Shimada H, Matsubara H, Shiratori T, et al: Phase I/II adenoviral p53 gene therapy for chemoradiation-resistant advanced esophageal squamous cell carcinoma. Cancer Sci 97: 554-561, 2006.

2. Edelman $\mathbf{J}$ and Nemunaitis $\mathrm{J}$ : Adenoviral p53 gene therapy in squamous cell cancer of the head and neck region. Curr Opin Mol Ther 5: 611-617, 2003.

3. Wolf JK, Bodurka DC, Gano JB, et al: A phase I study of Adp53 (INGN 201; ADVEXIN) for patients with platinum- and paclitaxel-resistant epithelial ovarian cancer. Gynecol Oncol 94: 442-448, 2004.

4. Oda K, Arakawa H, Tanaka T, et al: p53AIP1, a potential mediator of p53-dependent apoptosis, and its regulation by Ser-46-phosphorylated p53. Cell 102: 849-862, 2000.

5. Yoshida K, Monden M, Nakamura Y and Arakawa H: Adenovirusmediated p53AIP1 gene transfer as a new strategy for treatment of p53-resistant tumors. Cancer Sci 95: 91-97, 2004.
6. Matsuda K, Yoshida K, Taya Y, Nakamura K, Nakamura Y and Arakawa H: p53AIP1 regulates the mitochondrial apoptotic pathway. Cancer Res 62: 2883-2889, 2002.

7. Lunghi P, Costanzo A, Levrero $M$ and Bonati A: Treatment with arsenic trioxide (ATO) and MEK1 inhibitor activates the p73-p53AIP1 apoptotic pathway in leukemia cells. Blood 104: 519-525, 2004.

8. Wesierska-Gadek J, Gueorguieva M and Horky M: Roscovitineinduced up-regulation of p53AIP1 protein precedes the onset of apoptosis in human MCF-7 breast cancer cells. Mol Cancer Ther 4: 113-124, 2005.

9. Xie P, Tian C, An L, et al: Histone methyltransferase protein SETD2 interacts with p53 and selectively regulates its downstream genes. Cell Signal 20: 1671-1678, 2008.

10. Yagi S, Oda-Sato E, Uehara I, et al: 5-Aza-2'-deoxycytidine restores proapoptotic function of p53 in cancer cells resistant to p53-induced apoptosis. Cancer Invest 26: 680-688, 2008.

11. Gao C, Nakajima T, Taya Y and Tsuchida N: Activation of p53 in MDM2-overexpressing cells through phosphorylation. Biochem Biophys Res Commun 264: 860-864, 1999.

12. Tegeder I, Grosch S, Schmidtko A, et al: G protein-independent G1 cell cycle block and apoptosis with morphine in adenocarcinoma cells: involvement of p53 phosphorylation. Cancer Res 63: 1846-1852, 2003.

13. Sawaya M, Yoshimura T, Shimoyama T, Munakata A and Fukuda S: Difference of p53AIP1 mRNA expression in gastric mucosa between patients with gastric cancer and chronic gastritis infected with Helicobacter pylori. J Clin Gastroenterol 42: 351-355, 2008.

14. Yamashita SI, Masuda Y, Yoshida N, et al: p53AIP1 expression can be a prognostic marker in non-small cell lung cancer. Clin Oncol (R Coll Radiol) 20: 148-151, 2008.

15. Li XY, Liu JH, Li ZJ, et al: Construction of a recombinant adenoviral vector carrying integrin-alpha and the influence of integrin-alpha on the adhesion characteristics of SMMC-7721 cells. Zhongguo Wei Zhong Bing Ji Jiu Yi Xue 18: 413-416, 2006.

16. Zhao M, Xiao SW, Yang JX, Zhang SW and Lu YY: Detection of p53 gene change and serum antibody level in phase II clinical trial of ad p53 gene therapy. Zhonghua Yi Xue Za Zhi 85: 3495-3498, 2005.

17. Momand J, Zambetti GP, Olson DC, George D and Levine AJ: The mdm-2 oncogene product forms a complex with the p53 protein and inhibits p53-mediated transactivation. Cell 69: 1237-1245, 1992.

18. Kussie PH, Gorina S, Marechal V, et al: Structure of the MDM2 oncoprotein bound to the p53 tumor suppressor transactivation domain. Science 274: 948-953, 1996.

19. Bouska A, Lushnikova T, Plaza S and Eischen CM: Mdm2 promotes genetic instability and transformation independent of p53. Mol Cell Biol 28: 4862-4874, 2008.

20. Clegg HV, Itahana $\mathrm{K}$ and Zhang Y: Unlocking the Mdm2-p53 loop: ubiquitin is the key. Cell Cycle 7: 287-292, 2008.

21. Zhang Z and Zhang R: Proteasome activator PA28 gamma regulates $\mathrm{p} 53$ by enhancing its MDM2-mediated degradation. EMBO J 27: 852-864, 2008.

22. Weber JD, Taylor LJ, Roussel MF, Sherr CJ and Bar-Sagi D: Nucleolar Arf sequesters Mdm2 and activates p53. Nat Cell Biol 1: 20-26, 1999.

23. Honda R and Yasuda H: Association of p19(ARF) with Mdm2 inhibits ubiquitin ligase activity of Mdm2 for tumor suppressor p53. ЕMBO J 18: 22-27, 1999. 